\title{
Comparative analysis on the applicability of evaluation method for water resources carrying capacity
}

\author{
Qin Jing ${ }^{1}$, Wu Zhi-wang ${ }^{2}$, Feng Jie ${ }^{1, a}$, Niu Wen-long ${ }^{1}$ \\ ${ }^{1}$ China Institute of Water Resources and Hydropower Research, Beijing 100044, China; \\ ${ }^{2}$ Hohai University, Nanjing 210098, China
}

\begin{abstract}
Based on the analysis and summary of the origin and definition contents of water resources carrying capacity, this paper analyzes the applicability of water resources carrying capacity evaluation method from the view of quantity and quality of Evaluation index selection, index weight ratio, vertical and horizontal comparison of water resources carrying capacity, dynamic prediction, evaluation result form and regulation, and prospects and analyses from the perspectives of the perfections on water resources carrying capacity definition, the researches on applicable method and Its application and so on.
\end{abstract}

\section{Introduction}

The rapid development of China's economy and society and the significant improvement in the living standards of urban and rural residents have witnessed increasing problems such as water shortage, water ecological damage and water environment pollution. Under the influence of global climate change, extreme anomalies in the region are frequent, and China's economy and society are facing serious problems such as water security, resource security and environmental safety. In order to ensure the safety of water resources in countries and regions, the Third Plenary Session of the 18th Central Committee of the Communist Party of China clearly stated that it is important to establish a monitoring and early warning mechanism for resource and environmental carrying capacity, and to implement restrictive measures for water and soil resources, environmental capacity and marine resources overloaded areas. The series of policy documents issued afterwards emphasized that it is necessary to strictly abide by the ecological red line of resources and environment, implement the most stringent water resources management system, and strengthen the rigid constraints of water resources carrying capacity in regional development and industrial layout, thereby promoting economic and social development and water resources conditions to adapt.

Water resources carrying capacity is a comprehensive indicator that comprehensively reflects the ability of water resources to support the social economy and the ecological environment. It is also an important measure of regional sustainable development and industrial optimization. The purpose of research is to make "water resources - social environment - ecological environment "coordinated green development. Therefore, the study of water resources carrying capacity can provide a scientific basis for optimizing the allocation of regional water resources ${ }^{[1]}$ and improving water use efficiency. At the same time, it has important practical guiding significance for the rapid development of industrialization, the improvement of people's living standards and the realization of regional sustainable development. This paper focuses on the inductive analysis of the definition and connotation of water resources carrying capacity and the comparative analysis of the applicability of evaluation methods, so that the research and application of water resources carrying capacity can play a better role.

\section{Definition and connotation of water resources carrying capacity}

\subsection{The origin of research on water resources carrying capacity}

The idea of carrying capacity first appeared in the Aristotle era ${ }^{[2]}$, and was later applied to physical mechanics to characterize the maximum load that a loadbearing object can withstand while maintaining its own state of damage. The water resources carrying capacity is the specific application of bearing capacity in the field of water resources ${ }^{[3]}$. Throughout the quantitative research origin and development process of water resources carrying capacity, it can be roughly divided into three stages (Figure 1).

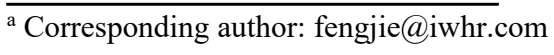




\begin{tabular}{|c|c|c|c|}
\hline $\begin{array}{l}\text { important } \\
\text { events }\end{array}$ & $\begin{array}{l}\text { Malthus }{ }^{[4]} \text { suggested that food would } \\
\text { limit the number of people, Verhulst } \\
\text { further proposed the logarithmic } \\
\text { growth equation }{ }^{[2]} \text { to quantitative } \\
\text { such a restriction, Park et al. }{ }^{[5]} \text { first } \\
\text { introduced the concept of carrying } \\
\text { capacity in human ecology }\end{array}$ & $\begin{array}{l}\text { Meadows et al. }{ }^{[9]} \text { proposed the } \\
\text { concept of "limit of Growth". } \\
\text { UNESCO, FAO and other } \\
\text { organizations }{ }^{[7-8]} \text { jointly carried out } \\
\text { a series of research on carrying } \\
\text { capacity project }\end{array}$ & $\begin{array}{l}\text { In the study of carrying } \\
\text { capacity of water resources } \\
\text { and development strategies } \\
\text { and countermeasures in } \\
\text { Xinjiang, Chinese scholars put } \\
\text { forward the issue of "water } \\
\text { resources carrying capacity" for } \\
\text { the first time }{ }^{[9]} \text {. }\end{array}$ \\
\hline stages & $\begin{array}{l}\text { The idea, definition and quantification } \\
\text { of resource carrying capacity begin to } \\
\text { sprout }\end{array}$ & $\begin{array}{l}\text { The definition of carrying capacity and } \\
\text { the quantitative method are initially } \\
\text { formed and used in practice }\end{array}$ & $\begin{array}{l}\text { Preliminary establishment of } \\
\text { definition and quantitative } \\
\text { method of water resources } \\
\text { carrying capacity }\end{array}$ \\
\hline \multicolumn{2}{|c|}{ The 1780 s } & \multicolumn{2}{|c|}{ The late $1980 \mathrm{~s}$} \\
\hline aracteristics & $\begin{array}{l}\text { The study focuses on the field of } \\
\text { biology and human ecology, generally } \\
\text { characterizing the maximum or } \\
\text { "moderate" quantity of a species or } \\
\text { population in a particular } \\
\text { "environment" at a certain stage. }\end{array}$ & $\begin{array}{l}\text { In the context of global resource and } \\
\text { environment crisis, the research of } \\
\text { carrying capacity begins is inclined to } \\
\text { the resource and environment. Many } \\
\text { theoretical and practical results have } \\
\text { been produced }\end{array}$ & $\begin{array}{l}\text { Combined with the sustainable } \\
\text { development theory, the research } \\
\text { direction of water resources } \\
\text { carrying capacity is systematic and } \\
\text { comprehensive }{ }^{[10] .}\end{array}$ \\
\hline
\end{tabular}

Fig 1. The origin of research on water resources carrying capacity

\subsection{Inductive analysis of the definition of water resources carrying capacity}

International research on water resources carrying capacity usually follows the idea and direction of resource carrying capacity, and further integrates it into sustainable development and comprehensive water resources management. Therefore, it pays great attention to the development and utilization of water resources and its limits ${ }^{[10-13]}$. The definition of water resources carrying capacity concept by Chinese scholars is more based on the water resources carrying capacity and its connotation. According to the research focus, the definition of water resources carrying capacity is also different. Based on the analysis of a large number of literatures, this paper analyzes the development and trends of water resources carrying capacity in a horizontal and vertical comparison.

The definition of water resources carrying capacity is still in the stage of in-depth development and improvement. From the perspective of horizontal development, this definition can be distinguished from narrow and broad sense. The definition of water resources carrying capacity in a narrow sense is generally

based on the capacity of water resources development ${ }^{[14-15]}$, the number of people supported, the scale of economic development supported, and the scale of the environment to be protected. Some definitions of this kind are from the perspective of the bearer body, and some are analyzed from the perspective of carrying the object, but the directions are relatively clear, so it is easy to quantify and analyze, which is suitable for exploring the maximum development level carried by the region; the definition of water resources carrying capacity in a broad sense is generally based on some kind of "abstract support ability". This "abstract support ability" can refer to the number of people supported and the scale of economic development supported ${ }^{[16-17]}$ and the ability to support the scale of a single target or the ability to support a comprehensive goal. This type of concept highlights the dynamics and interaction of water resources. It emphasizes the optimal dynamic development level of regional water resources, which is applicable to the dynamic, integrated and systematic research of water resources carrying capacity.

From the perspective of the vertical development of the definition of water resources carrying capacity, the definition of water resources carrying capacity is clearly proposed by Shi Yafeng et al. ${ }^{[14]}$ for the first time. This definition is based on "the largest population and the scale of social and economic development". Xu Youpeng [18] defined the water resources carrying capacity given by the maximum development capacity of water resources, and clarified the maximum supporting capacity of water resources, and also reflected the scale of the largest social and economic development to be carried. This definition is biased towards the quantification of water resources carrying capacity and explores the maximum carrying level. With the deep integration of water resources carrying capacity and sustainable development theory, the definition of water resources carrying capacity emphasizes the sustainable carrying capacity as the principle, and the moderate carrying capacity of maintaining the ecological environment as the condition, and the reasonable carrying capacity after reasonable optimization and configuration. With the continuous systematic and integrated research on water resources carrying capacity, the definition of water resources carrying capacity has been further improved. First, the ecological environment is gradually taken as the carrying object rather than the carrying condition. Some studies not only define the water resources carrying capacity from the water resources development capacity or the population 
quantity and economic development scale, but also define the water resources carrying capacity from the perspective of protecting the ecological environment; Second, the water resources carrying capacity itself also pays more attention to sustainable development, emphasizing the comprehensive benefits of water resources carrying capacity, and paying more attention to coordination and systematic development among the various carrying objects. The population is often based on the "sustainable average level of utility per capita". On a certain level, Long Tengrui et al. ${ }^{[19]}$ also proposed the definition of water ecology as the main body of the load, strengthened the ecological benefits of the water ecosystem, and further integrated the idea of sustainable development. Wang Jianhua et al. ${ }^{[20]}$ also proposed that water resources carrying capacity should be comprehensively considered from the aspects of water quantity, water quality, water space and water flow status.

In general, the research object and target of water resources carrying capacity are different, and the angles of definition are different. However, some definitions consider the deeper and systematic factors. Due to the limitations of relevant theories and quantitative methods at the current stage, there is a lack of corresponding systematic argumentation and practice test. With the cross-integration research with other disciplines, the introduction and application of new ideas, new technologies and new models, the definition of water resources carrying capacity will be continuously enriched and improved.

\subsection{The connotation of water resources carrying capacity}

The connotation of water resources carrying capacity is based on the conceptual analysis of water resources carrying capacity, and further explores the intrinsic attributes of water resources carrying capacity. It covers various aspects of water resources system, social economy, ecological environment and its interaction. It is roughly summarized as: time and space connotation, socio-economic connotation, ecological connotation and sustainable connotation $[19,21-22]$. On the whole, the definition of water resources carrying capacity is still a concept of extended ambiguity and connotation chaos ${ }^{[21]}$, and the connotation of water resources carrying capacity will be continuously enriched and improved.

\subsection{The evaluation index system}

In fact, the selection of the evaluation index system is related to the definition and connotation of the water resources carrying capacity and the evaluation method adopted. It not only reflects the characteristics of the water resources system itself, but also appropriately considers the reaction of the host object to the water resources carrying capacity and the interaction between the two. While comprehensively reflecting the characteristics of water resources carrying capacity of the region, the indicator system grasps the main influencing factors and main contradictions of the problems, and also accurately and sensitively reflects the dynamic changes of the carrying system.

\section{Comparative analysis of applicability of evaluation methods}

There are many methods for studying the water resources carrying capacity, but different methods have their advantages and disadvantages and scope of application and it is impossible to establish a unified evaluation model. Therefore, there is a great theory and practice for the comparative analysis of the applicability of water resources carrying capacity evaluation methods. At present, the evaluation methods can be generally classified into three categories: empirical formula method, which mainly includes the conventional trend method ${ }^{[2,3]}$, the quota analysis method ${ }^{[20,23]}$ and the background analysis method; the index system evaluation method mainly includes the fuzzy comprehensive evaluation method. ${ }^{[2,3,21]}$, principal component analysis $[2,21,23]$ and projection pursuit method; complex system analysis methods, including system dynamics [2, 3, 20], multi-objective analysis [2, 21] and "pressure-state-response (PSR)" models. The following is a comparative analysis of the applicability of the commonly used evaluation methods in the three types of methods.

In terms of the quantity and quality of evaluation indicators, the fuzzy comprehensive evaluation method generally determines the weight based on the analytic hierarchy process.

If there is a large amount of repetitive and redundant information in the selected evaluation indicators, even if the evaluation matrix is passed the consistency test after correction, this situation often leads to the amplification of the importance of the main influence factors, and the relatively minor evaluation factors is relatively weakened in theory, thereby the obtained comprehensive index value will be greater than the actual water resource carrying capacity evaluation index value. Therefore, when using this method to select indicators, it is generally necessary to control the number of indicators and select them from different angles, so as to avoid duplication and redundancy of information, and to highlight the main influencing factors and levels. Principal component analysis is based on the main influencing factors that are unrelated in many indicators. Therefore, the selection of indicators is relatively loose, basically only requiring the indicator system to fully reflect the status of water resources carrying capacity. The evaluation index of multi-objective analysis method is reflected in the determination of decision variables. The planning objectives and the set of constraints are linear or nonlinear functions of decision variables. The constraints often restrict and influence each other. The coupling effect of various constraints is often unsatisfactory ${ }^{[2]}$, and the coupling between the planning objectives and the constraints is difficult to grasp. The more multi-objective optimization models with more dimensions, the easier it is to find the local optimal 
results. This method is applicable to smaller model studies, and the constraint values do not require accurate predictions, only the range of upper and lower limits is determined. The system dynamics law requires a large number of data and evaluation indicators to build models, especially when constructing flow charts and their boundary conditions, the data demand is very large. There are also many single-index evaluation methods, such as the quota analysis method and the conventional trend method, which are generally based on the balance of supply and demand of water resources, and select one or several independent carrying factors to estimate the water resources carrying capacity.

In terms of the index weighting rate, the fuzzy comprehensive method takes the analytic hierarchy process as an example, based on the maximum eigenvalue of the judgment matrix and its eigenvector. If the judgment matrix satisfies the consistency test, the feature vector is taken as a weight vector, thereby determining the distribution of the weight coefficients. Due to the strong degree of human interference in the judgment matrix, the weight determined by the rate is less objective; the principal component analysis rule is based on a correlation matrix composed of a multi-year time sample sequence or a sample sequence of each subregion, and obtains the eigenvalues and eigenvectors of the correlation matrix. The contribution rate is obtained according to the eigenvalue, and the corresponding principal component load matrix is obtained to determine the weight of each factor in each principal component, and the weight ratio is determined to be objective; the multi-objective optimization method indirectly determines the weight of the decision variable by changing the weight and priority of the objective function. The weight of the method is actually determined by the multi-objective scheme, and the optimization analysis processing of each scheme can be performed, as well as the comparative analysis of each scheme.

If the system dynamics method predicts the water resources carrying capacity based on the current situation, it is generally simulated according to the similar change rules of historical data, and then the water resources carrying capacity evaluation is carried out by some multi-objective index system method or some comprehensive evaluation indicators. If the comparison is made between different water resources optimization allocation schemes, the weighting rate is reflected in the adjustment of the parameters of some models and the internal feedback relationship of the system, which is greatly influenced by the decision value orientation. Therefore, complex system analysis is more suitable for multi-objective system decision-making problems.

Broadly speaking, the research on longitudinal comparative analysis of water resources carrying capacity can provide more methods for longitudinal comparative analysis of water resources carrying capacity. In the empirical formula method, the vertical comparison of water resources carrying capacity is generally based on the single factor loading level or the water supply and demand situation. Due to insufficient consideration of the factors affecting water resources carrying capacity, it is difficult to comprehensively and vertically analyze the water resources carrying capacity; Both the fuzzy comprehensive evaluation method and the principal component analysis method can compare the water resources carrying capacity in a reasonable longitudinal direction. Both of them are longitudinal analysis of water resources carrying capacity while maintaining the weight of each influencing factor. The difference is that the fuzzy comprehensive evaluation rule is more biased towards the static evaluation of water resources carrying capacity, and the water resources carrying capacity of each stage is evaluated one by one. However, the principal component analysis rule is based on the correlation matrix analysis of various index sequences for many years. The results of water resources carrying capacity evaluation at each stage are given by comprehensive scoring method, which is relatively intuitive, and even has the classification of classification and sorting. The complex system analysis rule is more inclined to the dynamic development of the system, and is suitable for studying the internal hierarchy and dynamic prediction of the system. For example, the biggest advantage of the system dynamics method is to establish a perfect feedback system within the system, reasonably predict the decision variables according to different drafting schemes, and perform longitudinal analysis on the existing index data of each stage, while the complex system analysis method is not applicable.

In the horizontal comparative analysis of water resources carrying capacity, which is similar to the longitudinal comparative analysis of water resources carrying capacity, so only the fuzzy comprehensive evaluation method and principal component analysis method are compared here: Since the fuzzy comprehensive evaluation method does not remove the repeated part of the indicator information, it is easy to cause the repeated information to be repeatedly calculated, which actually increases the weight of some influencing factors, and the weight of the influencing factors that can truly reflect the differences of each subarea is often mitigated. It may lead to the disorder of the relative capacity of water resources in similar subregions, which also puts higher requirements on the selection of the index system; principal component analysis can generally eliminate the duplicate information between principal components while maintaining most of the data, and the operation is more efficient, which is suitable for horizontal comparative analysis of water resources carrying capacity.

It is worth mentioning that the vertical and horizontal comparative analysis of water resources carrying capacity is based on the data of sufficient indicators, especially the longitudinal comparison of water resources carrying capacity requires long time series data. However, it is difficult to analyze due to lack of data in many areas, so it is recommended to use background analysis to analyze the areas similar to their water resources carrying capacity.

In the dynamic prediction of water resources carrying capacity, the empirical formula method has fewer predictive indicators, and ignores the interrelationship between each load factor, so it can predict the water 
resources carrying capacity simply and sensitively. For example, according to the conventional trend method, the corresponding carrying population or socioeconomic scale can be calculated based on the predicted amount of exploitable water, the proportion of water used in each water sector, agricultural and social watersaving indicators, and relevant indicators of carrying factors. When the index system evaluation method is used for dynamic prediction, the prediction of the index value is often based on a certain planning or empirical formula. Although there is a lack of certain systemicity, the short-term prediction results have strong practicability. The quantitative and qualitative dynamic prediction of water resources carrying capacity of complex system analysis method pays more attention to systemicity and integrity. For example, the system dynamics method not only needs to fit the change law similar to the historical data, but also takes into account the development trend of the system, which may lead to the proposed parameters not reflecting the current state of water resources carrying capacity. And with the changes of the system, the proposed parameters are difficult to accurately predict the state of long-term water resources carrying capacity, especially when using nonlinear equations, small information disturbances may lead to large changes in the evaluation results. Therefore, the method is suitable for short-term and medium-term dynamic prediction of water resources carrying capacity.

In terms of the evaluation results of water resources carrying capacity, such as the fuzzy comprehensive evaluation method, principal component analysis method and the comprehensive index method, the index system evaluation method generally judges the water resources carrying capacity by a certain comprehensive evaluation score, which can be roughly judged by the level of water resources carrying capacity, such as overload, criticality and non-overloading. In addition, principal component analysis can also rank the various levels and their internal water carrying capacity. Complex system analysis methods such as multi-objective analysis, system dynamics and extension matter-element models can explore the water resources carrying capacity of the optimal state of the system according to a certain planning state. The results of the multi-objective analysis method are biased towards the balance of comprehensive benefits, and the results of the system dynamics method are mainly reflected in the development trend of decision variables through various programs and the supply and demand of water resources, so as to comprehensively analyze the water resources carrying capacity. The empirical formula method such as the conventional trend method and the quota analysis method generally calculates the maximum carrying capacity of the regional water resources theory based on the single-entry factor.

In the regulation of water resources carrying capacity, the empirical formula method is difficult to reflect the interaction and connection between the "water resources - economic society - ecological environment" system, which leads to low accuracy of dynamic load calculation of system changes, and it is difficult to systematically support water resources carrying capacity regulation program; the principal component analysis method and the fuzzy comprehensive rule are based on the main driving factors of water resources carrying capacity and the bearing degree of each factor, but they are biased towards qualitative. Model methods such as system dynamics can determine the main sensitive variables based on the sensitivity analysis, and thus propose more various control schemes. And through the simulation operation simulation, based on the decision variables and the overall target optimization quantitative analysis, the various control schemes are selected. In general, the more comprehensive, detailed, and quantitative decisionmaking variables or evaluation indicators can support the specific measures and detailed planning for the development of control programs.

The above is mainly to select some common methods from the three major methods for comparative analysis of applicability, mainly to provide ideas for method comparison. It is not refined to each method, so it is inevitable that there will be some omissions in the applicability of some methods.

\section{Summary and prospect}

Through the summary analysis of the definition of water resources carrying capacity and the comparative analysis of the applicability of water resources carrying capacity research methods, the following summary and prospects are further obtained from the research and practical application of well-defined and applicable methods.

(1) The definition and connotation of water resources carrying capacity at the present stage are still in the stage of deep development. Many academic and technical problems also restrict the enrichment, promotion and application of the definition of water resources carrying capacity. The definition and connotation of water resources carrying capacity development in the future needs to be based on the analysis of existing achievements and related theoretical systems, and continuously strengthen the comprehensive research of cross-integration with other disciplines, bold innovation, especially the introduction of new theories and new ideas. Only when the basic theory is perfect can we establish a more effective, comprehensive and comprehensive evaluation index system and quantitative model method.

(2) Comparative analysis of applicability of evaluation methods is essential for the application and promotion of water resources carrying capacity. There are various methods for evaluating the water resources carrying capacity. Each method has its own characteristics and applicability. Thus, choosing the appropriate method is the basis for ensuring accurate evaluation results. In addition, in order to better propose and improve the evaluation method, it is necessary to pay attention to the combination with some new theories and new technologies. Big data mining techniques such as the current widely used technology have been practiced in the field of hydrology. With the intensification of human activities and the impact of climate change ${ }^{[24]}$, population patterns, industrial patterns, water use patterns, hydrological cycles, water 
environment ${ }^{[25]}$, and water control ideas have undergone profound changes. In addition, the factors considered by water resources are more complex and far-reaching. How to extend this technology to the assessment of water resources carrying capacity, especially in the era of water conservancy information, has a wide application prospect.

(3) In recent years, various major industries have successively carried out research on water resources carrying capacity evaluation and industrial optimization layout adjustment measures. Different from traditional watershed, regional or urban water resources carrying capacity research, many major industries have regional dispersion and rapid development, so the horizontal and vertical comparisons are characterized by large differences.

When studying the water resources carrying capacity in the region, we have to take into account the impact of the development of the industry on the regional water resources carrying capacity, and also propose the industrial optimization adjustment based on the evaluation results of water resources carrying capacity, thus reflecting sustainable green development and the idea of "Deciding production by water". The research on water resources carrying capacity of major industries is more practical and will become the focus and hotspot of future research, which will further enrich and improve the relevant theories of water resources carrying capacity.

Taking the assessment of water resources carrying capacity of Xinjiang Textile Industry Base and the adjustment strategy of industrial optimization layout as an example, we found that the industry is distributed in multiple regions, and the available data in some regions is very scarce. In order to comprehensively analyze the water resources carrying capacity of the Xinjiang textile industry base, according to the above-mentioned comparative analysis of the applicability of the water resources carrying capacity evaluation method, it is difficult for a single traditional evaluation method to fully achieve these goals. Therefore, it is necessary to consider a new method or a combination of methods to analyze the water resources carrying capacity of the Xinjiang textile industry base and propose an effective industrial layout optimization adjustment strategy. We consider joint modeling by using a variety of methods, such as using principal component analysis and multiobjective optimization analysis to build models. Principal component analysis can be used to analyze the status and main driving factors of water resources carrying capacity in different regions. It can also longitudinally analyze the changing trend of water resources carrying capacity in the region where the industrial base is located and the impact of the development of the textile industry on the carrying capacity of water resources. Through this method, the main driving factors of water resources carrying capacity in the region where the textile industry base is located are roughly determined. Using these drivers as decision variables, combined with the proposed water resources optimization configuration plan, we construct a multiobjective optimization model to further verify the evaluation results. It is determined that these main driving factors are based on the optimal quantitative scope based on the sustainable development of water resources carrying capacity, so as to propose corresponding industrial layout optimization strategies more specifically.

\section{Acknowledgement}

This work was supported by the Ministry of Water Resources budget project for 2018(SJ0149B222018).

\section{References}

1. Xuebin Q, Zhongdong H, Dongmei Q, et al. Research progress on rational allocation of water resources in irrigated area. J. Progress in water Science, 26, 2: 287-295 (2015).

2. Lijuan D, Yong X. Research progress and revelation of water resources carrying capacity. $\mathrm{J}$. Soil and water Conservation Studies, 22, 3: 341-348 (2015).

3. Qingchun D, Changming L, Xiaonan C,Wenhua L,Hongxing Z. Discussion on the concept and research method of regional water resources carrying capacity. J. Geography, 65, 1: 82-90 (2010).

4. Malthus T R. An eassy on the principle of population.London;Pickcring, (1798).

5. PARK R E, BURGESS E W. Introduction to the science of sociology. M. Chicago: University of Chicago Press, (1921).

6. MEADOWS D H, RANDERS J, et al.The limits to growth. M. New York, (1972).

7. Higgins G M,Kassam A H,Naiken L,et al.Potential population supporting capacities of lands in the developing world. R. Roma:FAO,(1982).

8. UNESCO,FAO.Carrying capacity assessment with a pilot study of Kenya: A resource accounting methodology for sustainable development. Paris and Rome, (1985).

9. Xinjiang Water Resources Soft Science research group. Water resources in Xinjiang and its carrying capacity and development strategy. J. Water Conservancy and hydroelectric Technology, 9: 2-9 (1989).

10. Hongliang Z, Bo H. The theoretical basis of bearing capacity analysis from the properties of bearing capacity. J. China's land and resources economy, 26, 8: 57-60 (2013).

11. Peterson D H. Flooda keys carring capacity study. J. Proceedings of the Water Environment Federatioon, 13:489-501 (2013).

12. Harris Jonathan $\mathrm{M}$, et a1. Carrying capacity in Agriculture : Globe and regional issue. Ecological Economics. J. 129, 3: 443461 (1999) . 
13. Rijisberman. Different approaches to assessment of design and management of design and management of sustainable urban water system. J. Environment Impact Assessment Review. 129, 3: $333 \sim 345$ (2000).

14. Yafeng S, Yaoguang Q. Water resources carrying capacity and its rational utilization in Urumqi river Basin. M. Beijing: Science Press, 94-111 (1992).

15. Jun X, Yizhong Z. Measurement of water resources security: Research and challenge of water resources carrying capacity. J. Journal of Natural Resources, 3: 262-269 (2002).

16. Shangyou F, Guoquan L. Framework for the sustainable use of water resources. J. Progress in Water Science, 4: 2-8 (1997).

17. Yanghe H, Xiaohui J, Qiang H, et al. HUANG Qiang, Schollier. Two Yuan mode study on dynamic simulation model of water resources carrying capacity system J. Geography Studies, 2: 191-198 (2001).

18. XU Youpeng. Comprehensive evaluation of water resources carrying capacity in arid area--a case study of Hotan River basin in Xinjiang. J. Journal of Natural Resources, 3: 229-237. (1993).

19. Tengrui L, Wenchao J, Qiang H. New understanding of the connotation of water resources carrying capacity. J. Journal of Water Conservancy, 1: 38-45 (2004).

20. Jianhua $\mathrm{W}$, Dachuan $\mathrm{J}$, Weihua $\mathrm{X}$, et al. The theoretical basis of water resources carrying capacity: definition connotation and scientific problems. J. Journal of Water Conservancy, 48, 12: 1399-1409 (2017).

21. Yunhai Z, Limin P, Min D, et al. Review of regional water resources carrying capacity evaluation at home and abroad. J. Science and Management, 30, 3: 21-24. (2010).

22. Guohua Z, Guojing Z, Xiaoyue L, et al. Analysis of connotation and theory of water resources carrying capacity. J. Soil and water Conservation Studies, 6: 289-291+294 (2007).

23. Guang Y. Study on evaluation model of water resources carrying capacity in Manas He Basin. D. Shihezi University, (2009).

24. Qiting Z, Xiuyu Z. Study on dynamic bearing capacity of water resources under climate change. J. Journal of Water Conservancy, 46, 4: 387 395(2015).

25. Nan S, Xiaohong R, Jie F. Research progress in the definition of suitable width of water ecological barrier. J. Progress in water science, 23, 04: 581589 (2012). 